\title{
Summary of UHECR Composition Measurements by the Telescope Array Experiment
}

\author{
John Belz* ${ }^{\dagger}$ \\ University of Utah, Salt Lake City, Utah, USA \\ E-mail: belz@physics.utah.edu \\ for the Telescope Array Collaboration
}

\begin{abstract}
We study the chemical composition of ultrahigh energy cosmic rays primarily using the $X_{\max }$ technique. The reconstruction techniques use events either seen by two of the TA fluorescence detectors (stereo mode), or by one fluorescence detector (monocular mode), and one fluorescence detector and the TA surface detector (hybrid mode). Each technique has its own acceptance imprinted on the data. We compare the resulting $X_{\max }$ distributions to those of shower Monte Carlo simulations, by generating events and analyzing them with exactly the same programs as the data. For energies greater than $10^{18} \mathrm{eV}$, the results in all cases appear to be an unchanging light composition. In this talk, a summary of all TA $X_{\max }$ data will be presented.
\end{abstract}

The 34th International Cosmic Ray Conference,

30 July- 6 August, 2015

The Hague, The Netherlands

\footnotetext{
*Speaker.

${ }^{\dagger}$ Full author list: http://www.telescopearray.org/images/papers/ICRC2015-authorlist.pdf.
} 


\section{Introduction}

The precise composition of Ultra-High Energy Cosmic Rays (UHECR), coupled with their energy spectrum and arrival directions, can inform us as to their sources, acceleration mechanisms, and propagation through the galactic and extragalactic media.

The observation $[1,2,3]$ of a suppression in the cosmic ray flux at an energy of approximately $10^{19.72} \mathrm{eV}$ is readily understood in terms of the $\operatorname{GZK}[4,5]$ mechanism, provided the flux at the highest energies is dominated by protons arriving from cosmologically significant distances. In this case, the resonance photoproduction of pions in interactions with the cosmic microwave background (CMBR) places an effective horizon of order 100 Megaparsecs on the sources of UHECR arriving at Earth.

However if the UHECR composition is heavy or changing with energy, interpretation becomes more challenging. Nuclei will fragment when bombarded with CMBR gamma rays, generally at a threshold energy lower than that of the protonic energy loss process. The threshold energy grows with the mass of nuclei, and for heavier nuclei such as iron the fragmentation energy will be comparable to that of the observed flux suppression. However the most recent composition measurements indicate that a large iron component in the UHECR flux is unlikely $[6,7,8]$.

Alternative explanations to the observed suppression in the absence of a proton-dominated flux include acceleration limits of relatively nearby sources [9]. One might roughly expect that the maximum energy of a particular source $E_{\max } \approx Z E_{p}$, where $Z$ is the nuclear charge and $E_{p}$ is a source-dependent constant (the maximum proton energy). The signature of such a model would be an energy-dependent composition, and the fact that the largest suppression occurs close to the expected GZK energy would be merely coincidental.

\section{The Telescope Array Observatory}

Modern UHECR observatories which utilize the nitrogen fluorescence technique typically try to infer chemical composition by studying the distribution of air shower maximum or $X_{\max }$. This method makes use of the fact that heavier nuclei have a larger interaction cross section in the Earth's atmosphere. The Telescope Array (TA) Observatory (Figure 1) in Millard County, Utah, U.S.A. employs three fluorescence detectors [10] overlooking a $700 \mathrm{~km}^{2}$ surface detector array [11]. Air shower geometry is determined either by viewing the shower stereoscopically in fluorescence (stereo mode), or by using one or more fluorescence detectors in conjunction with the surface detector array (hybrid mode). Then, shower maximum is determined for showers individually from the fluorescence light profile.

Both stereo and hybrid mode accept air showers within a limited range of shower geometries, and hence impart a bias to the $X_{\max }$ distribution relative to the full $X_{\max }$ distribution of cosmic rays which strike the Earth. In Telescope Array, this bias is evaluated by comparing geometrical and $X_{\max }$ distributions to those of shower Monte Carlo simulations.

As of this writing, Telescope Array has published the results of one $X_{\max }$ analysis, that using fluorescence data collected by the Middle Drum Fluorescence Detector, and observed in hybrid mode with the TA surface detector [8]. See Figure 2. An update will be given at this conference $[13,14]$. Other analyses currently in progress with release anticipated by ICRC 2015 in- 


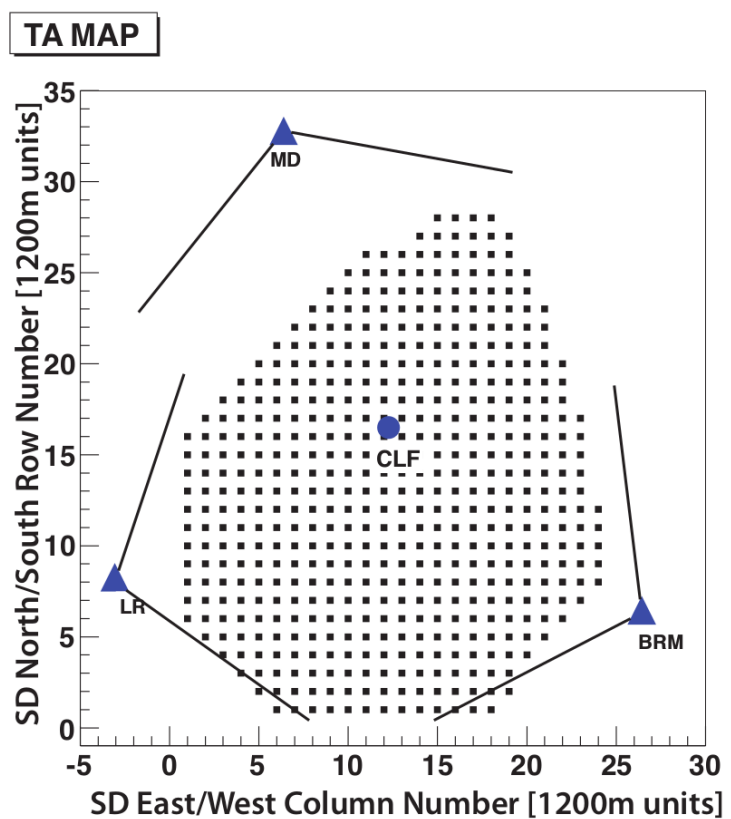

Figure 1: The Telescope Array detector configuration. The filled squares are the 507 surface detector (SD) scintillators on a $1.2 \mathrm{~km}$ grid. The SD scintillators are enclosed by three fluorescence detectors shown in filled triangles together with their field of view in solid lines. The northernmost fluorescence detector is called Middle Drum while the southern fluorescence detectors are referred to as Black Rock Mesa and Long Ridge. The filled circle in the middle equally spaced from the three fluorescence detectors is the Central Laser Facility used for atmospheric monitoring and detector calibration.

clude hybrid mode analyses featuring the Black Rock and Long Ridge fluorescence detectors [15], stereo mode analyses from the three possible pairings of TA fluorescence detectors [16], and finally monocular analysis using data from Black Rock and Long Ridge [17]. In addition, new results from a measurement of the proton-air cross section - calculated using the shape of the $X_{\max }$ distribution - will be presented at this conference [18].

\section{Conclusions}

An overview of the latest $X_{\max }$ results from Telescope Array will be presented at the $34^{\text {th }}$ International Cosmic Ray Conference.

\section{Acknowledgements}

The Telescope Array experiment is supported by the Japan Society for the Promotion of Science through Grants-in-Aid for Scientific Research on Specially Promoted Research (21000002) "Extreme Phenomena in the Universe Explored by Highest Energy Cosmic Rays" and for Scientific Research (19104006), and the Inter-University Research Program of the Institute for Cosmic Ray Research; by the U.S. National Science Foundation awards PHY-0307098, PHY-0601915, PHY-0649681, PHY-0703893, PHY-0758342, PHY-0848320, PHY-1069280, PHY-1069286, PHY1404495 and PHY-1404502; by the National Research Foundation of Korea (2007-0093860, R32- 

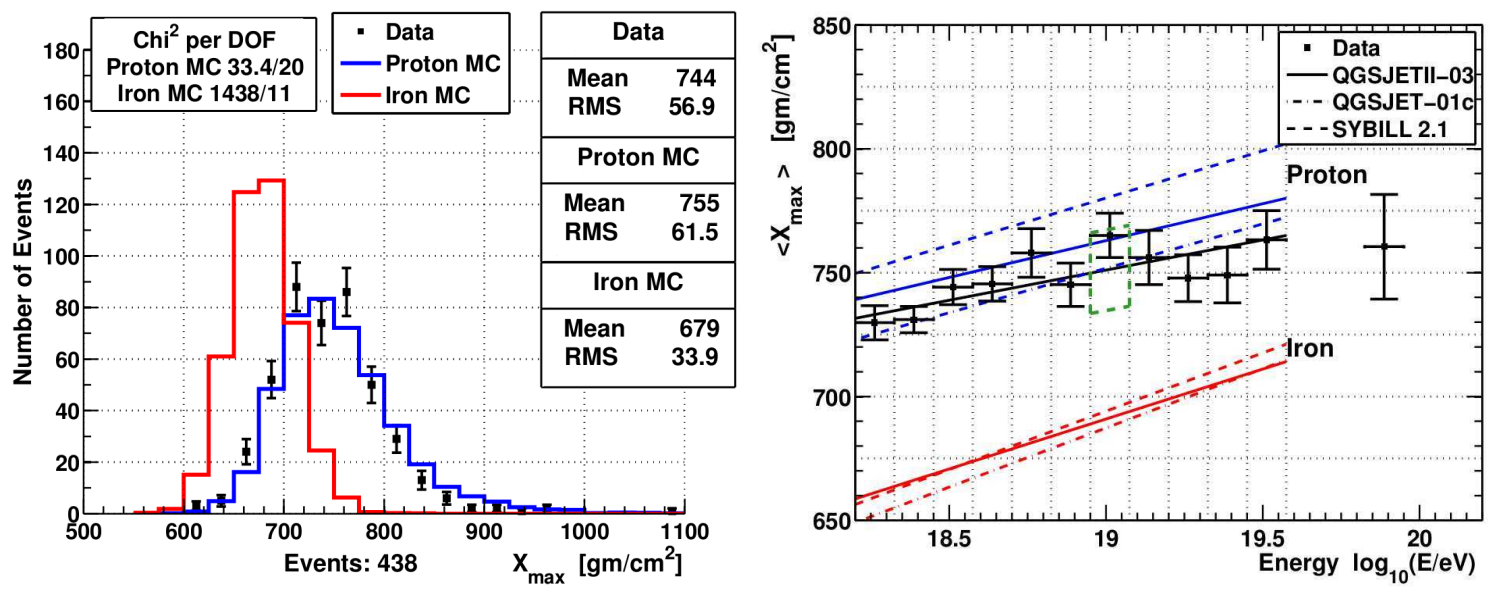

Figure 2: Left: Comparison of $X_{\max }$ distribution of TA Middle Drum hybrid data with QGSJETII-03 [12] Monte Carlo expectation for pure proton (blue) and pure iron (red) distributions. Right: Evolution of mean $X_{\max }$ versus energy, for TA MD hybrid data and various high-energy hadronic interaction models.

10130, 2012R1A1A2008381, 2013004883); by the Russian Academy of Sciences, RFBR grants 11-02-01528a and 13-02-01311a (INR), IISN project No. 4.4502.13, and Belgian Science Policy under IUAP VII/37 (ULB). The foundations of Dr. Ezekiel R. and Edna Wattis Dumke, Willard L. Eccles, and George S. and Dolores Doré Eccles all helped with generous donations. The State of Utah supported the project through its Economic Development Board, and the University of Utah through the Office of the Vice President for Research. The experimental site became available through the cooperation of the Utah School and Institutional Trust Lands Administration (SITLA), U.S. Bureau of Land Management, and the U.S. Air Force. We also wish to thank the people and the officials of Millard County, Utah for their steadfast and warm support. We gratefully acknowledge the contributions from the technical staffs of our home institutions. An allocation of computer time from the Center for High Performance Computing at the University of Utah is gratefully acknowledged.

\section{References}

[1] R. U. Abbasi et al., Phys. Rev. Lett. 100, 101101 (2008).

[2] J. Abraham et al., Phys. Rev. Lett. 101, 061101 (2008).

[3] T. Abu-Zayyad et al., Ap. J. Lett. 768:L1 (2013).

[4] T. Greisen, Phys. Rev. Lett. 16, 748 (1966).

[5] G. T. Zatsepin and V. A. Kuz'min, JETP Lett. 4, 78 (1966).

[6] R. U. Abbasi et al., Phys. Rev. Lett. 104, 161101 (2010).

[7] A. Aab et al., Phys.Rev. D90 12, 122005 (2014). A. Aab et al., Phys.Rev. D90 12, 122006 (2014).

[8] R. U. Abbasi et al. Astropart.Phys. 6449 (2014).

[9] R. Aloisio et al., Astropart. Phys. 34620 (2011). 
[10] H. Tokuno et al., Nucl. Instrum. Meth. A676 54 (2012).

[11] T. Abu-Zayyad et al., Nucl. Instrum. Meth. A689 87 (2012).

[12] S. Ostapchenko, Nucl. Phys. B, Proc. Suppl. 151, 143 (2006).

[13] J. P. Lundquist, Proceedings of the $34^{\text {th }}$ ICRC, Contribution \#1330.

[14] J. P. Lundquist, Proceedings of the $34^{\text {th }}$ ICRC, Contribution \#1332.

[15] D. Ikeda and W. Hanlon, Proceedings of the $34^{\text {th }}$ ICRC, Contribution \#0906.

[16] T. A. Stroman and Y. Tameda, Proceedings of the $34^{\text {th }}$ ICRC, Contribution \#0905.

[17] T. Fujii, Proceedings of the $34^{\text {th }}$ ICRC, Contribution \#0714.

[18] R. U. Abbasi, Proceedings of the $34^{\text {th }}$ ICRC, Contribution \#1095. 